\title{
Design of the Vehicular SINSIOD Positioning Based on Locality-Aware Auxiliary
}

\author{
Si Chen ${ }^{\text {a }}$, Qiyuan Zhong ${ }^{\mathrm{b}}$, Lilong Tan ${ }^{\mathrm{c}}$, Peng Wang ${ }^{\mathrm{d}}$, Yantao Zhang ${ }^{\mathrm{e}}$ \\ High and New Technology Institute, Xi' an, 710025, China. \\ a664561690@qq.com, bzhongqiyuan1225@163.com, c15349227983@189.cn \\ d1754979391@qq.com, e820289171@qq.com
}

Keywords: Navigation, RFID, Locality-Aware, SINS/OD, Positioning correction.

\begin{abstract}
In order to solve the problem of launching vehicles positioning and orientation technology when losing GPS aided,a navigation scheme based on radio frequency identification (RFID) to assist the SINS/odometer (OD) are proposed.A variety of ways to locate vehicle are designed based on RFID and the actual use of related problems are analyzed.And appliy it to the SINS/OD model, and then build the SINS/OD/RFID model. The model provides a new angle and new method of vehicle auxiliary position.Results show that the precision of the position scheme can reach meters, extend navigation time, meet the precision requirement, and improve the use efficiency
\end{abstract}

\section{Introduction}

Vehicle positioning and orientation technology of missile launcher is the key technology to realize the launch of missiles without preset positions, Essential refers to the vehicle navigation system in the process of launching vehicles in real time to provide accurate latitude and longitude, elevation, north azimuth and attitude angle of the aiming and launch missiles required for the basic information[1].Vehicle-based positioning and orientation systems based on combat applications require autonomy, speediness, accuracy and immunity to interference. SINS has been widely recognized by its complete autonomy and practicability, but due to the accumulation of errors over time, SINS cannot be used singly for long periods of time [2]. Due to its weakened vehicle maneuverability,initially zero-speed correction or preset point parking correction ways is not favored[3]. Although Using SINS/GPS integrated navigation system restrain the divergence of the error, but the GPS signal can be easily blocked and the technology is controlled by the United States, and therefore cannot be used in combat [4]. Even if the localization of BeiDou is used, similar problems exist, such as interference of satellite signals. similar effects are obtained by using map matching technology [5], but there is a high demand for map accuracy. Data processing Technology also has higher requirements and other issues; SINS/odometer (OD) integrated navigation using dead reckoning technology autonomy is good, the error divergence to a certain extent, However, due to wheel idling, slipping and other problem, errors are also large after long-time navigation [6], and cannot solve the problem field navigation system error out of range or failure to restart and cannot effectively get the initial geographic information of the actual needs.

In recent years, the rapid development of Internet of Things technology has led to the rapid promotion and application of RFID radio frequency identification devices. RFID uses radio frequency signals to transmit the information stored in the tags to the RFID reader. It is an emerging means of identification and location. It has the advantages of simple structure, long life, harsh environment, non-contact, fast, safe, accurate and cheap (Military tag 10 cents) and other advantages [7]. RFID military applications can be traced back to World War II, the US military used to identify Allied aircraft, military RF tags in recent years has been used in military logistics, military vehicle positioning and other fields. Combined with the advantages of SINS / OD combination positioning and orientation system commonly used in the present stage, it is expected to set up a radio frequency positioning system in the combat area to make up for its deficiencies and assist in vehicle positioning. 


\section{Car SINS/OD Integrated Navigation Analysis}

SINS has the autonomy, is the preferred equipment used in vehicle positioning, SINS high-precision gyro and table navigation solution, from the starting point for the cumulative position and attitude estimation [8], show in Figure 1. Short-time, SINS solution accuracy is particularly high, but due to the constant drift and random drift exists, long flight, the error continues to allowable range of error, positioning data loss of combat value. Therefore, at the stage, high-precision speed odmeters (10-4 m/s) are commonly used in research facilities to suppress the divergence of errors and extend the navigation and positioning time to meet the requirements of the requirements of the war standards, achieving good results.

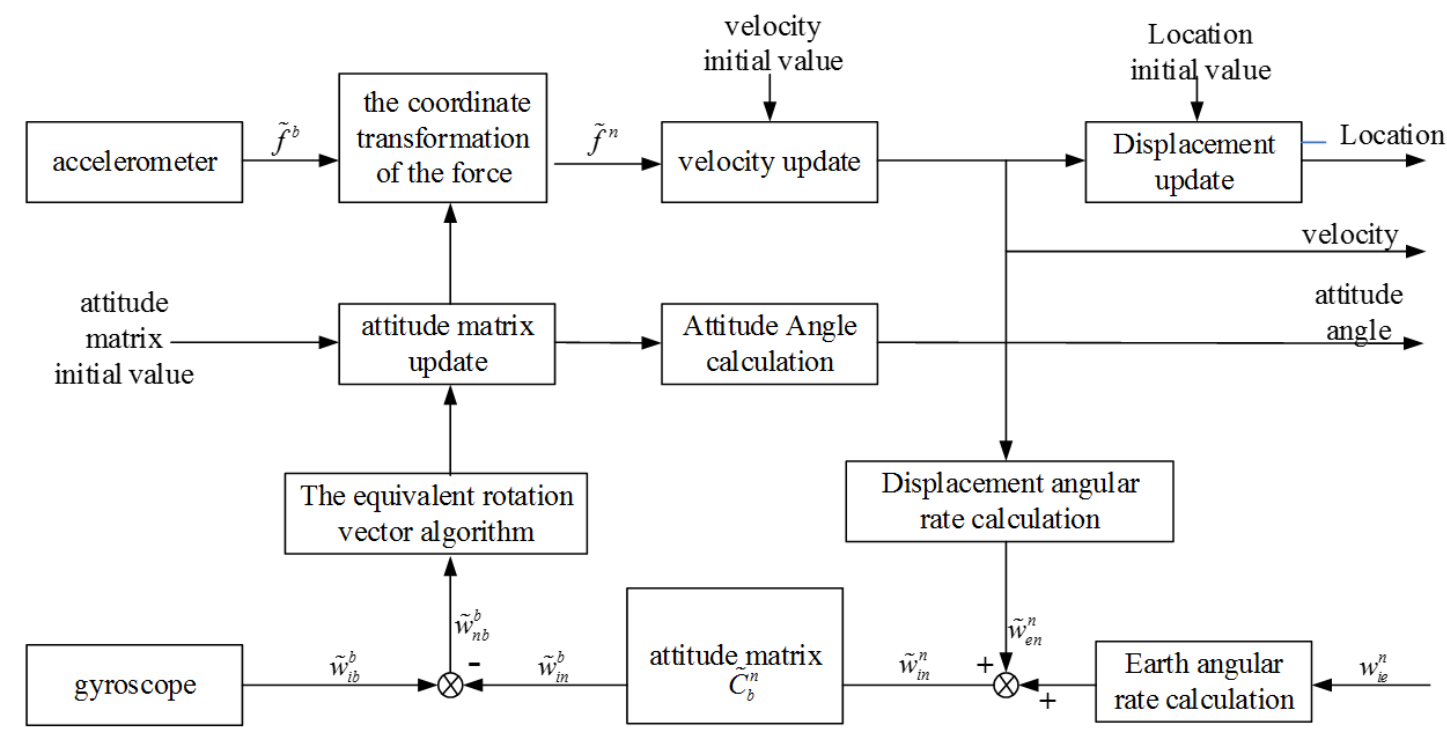

Fig.1 The strapdown inertial navigation calculation

\subsection{Inertial Guide and Odometer Error Analysis}

\subsubsection{Odometer Error Analysis}

Reference system selected East - North - Upward (ENU) Department of geography as a navigation coordinate system (n system), Inertial coordinate system and vehicle coordinate system identified coincidence (b system), The OD coordinate system (m system) is a right-front-upward right-handed rectangular coordinate system that is fixed to the vehicle body, So the theoretical output of OD in the m system can be expressed as:

$$
\mathbf{v}_{D}^{m}=\left[\begin{array}{lll}
0 & v_{D} & 0
\end{array}\right]^{\mathrm{T}}
$$

Because odometer scale factor error $\delta K_{D}$ exists, OD actual speed output $\tilde{\mathbf{v}}_{D}^{m}$ becomes:

$$
\tilde{\mathbf{v}}_{D}^{m}=\left(1+\delta K_{D}\right) \mathbf{v}_{D}^{m}=\left(1+\delta K_{D}\right)\left[\begin{array}{lll}
0 & v_{D} & 0
\end{array}\right]^{\mathrm{T}}
$$

Transformation to the n-system process, the use of the attitude matrix $C_{b}^{n}$ introduced SINS at this moment the attitude error, Get the output of OD under n series:

$$
\tilde{\mathbf{v}}_{D}^{n}=\tilde{C}_{b}^{n} \tilde{\mathbf{v}}_{D}^{m}=[I-\boldsymbol{\phi} \times] C_{b}^{n}\left(1+\delta K_{D}\right)\left[\begin{array}{lll}
0 & v_{D} & 0
\end{array}\right]^{\mathrm{T}}
$$

Reasonably discarding the high-order small quantities of and, expand to get:

$$
\tilde{\mathbf{v}}_{D}^{n} \approx C_{b}^{n} \mathbf{v}_{D}^{m}+\delta K_{D} C_{b}^{n} \mathbf{v}_{D}^{m}-(\phi \times) C_{b}^{n} \mathbf{v}_{D}^{m}
$$

At this point, the OD output speed error is derived as:

$$
\delta \mathbf{v}_{D}^{n}=\tilde{\mathbf{v}}_{D}^{n}-\mathbf{v}_{D}^{m}=\delta K_{D} \mathbf{v}_{D}^{n}+\boldsymbol{\phi} \times \mathbf{v}_{D}^{n}
$$

\subsubsection{SINS Error}

According to the literature [9], SINS error model for derivation and analysis of small misalignment angle is as follows. 


$$
\begin{aligned}
\dot{\boldsymbol{\phi}} & =\boldsymbol{\phi} \times \boldsymbol{w}_{i n}^{n}+\delta \boldsymbol{w}_{i n}^{n}-\delta \boldsymbol{w}_{i b}^{n} \\
& =\boldsymbol{\phi} \times \boldsymbol{w}_{i n}^{n}+\boldsymbol{M}_{2} \delta v^{n}+\left(\boldsymbol{M}_{1}+\boldsymbol{M}_{3}\right) \delta \boldsymbol{p}-\boldsymbol{\varepsilon}^{n}
\end{aligned}
$$

Attitude error:

$$
\begin{gathered}
\dot{\boldsymbol{\phi}}=\boldsymbol{\phi} \times \boldsymbol{w}_{i n}^{n}+\delta \boldsymbol{w}_{i n}^{n}-\delta \boldsymbol{w}_{i b}^{n} \\
=\boldsymbol{\phi} \times \boldsymbol{w}_{i n}^{n}+\boldsymbol{M}_{2} \delta v^{n}+ \\
\left(\boldsymbol{M}_{1}+\boldsymbol{M}_{3}\right) \delta \boldsymbol{p}-\boldsymbol{\varepsilon}^{n}
\end{gathered}
$$

Speed error:

$$
\begin{aligned}
\delta \dot{\boldsymbol{v}}^{n} & =-\boldsymbol{\phi} \times \boldsymbol{f}^{n}+\delta \boldsymbol{v}^{n} \times\left(2 \boldsymbol{w}_{i e}^{n}+\boldsymbol{w}_{e n}^{n}\right)+v^{n} \times\left(2 \delta \boldsymbol{w}_{i e}^{n}+\delta \boldsymbol{w}_{e n}^{n}\right)+\nabla^{n} \\
& =-\boldsymbol{\phi} \times \boldsymbol{f}^{n}+\delta \boldsymbol{v}^{n} \times\left(2 \boldsymbol{w}_{i e}^{n}+\boldsymbol{w}_{e n}^{n}\right)+\boldsymbol{v}^{n} \times\left(2 \boldsymbol{M}_{1} \delta \boldsymbol{p}+\boldsymbol{M}_{2} \delta \boldsymbol{v}^{n}+\boldsymbol{M}_{3} \delta \boldsymbol{p}\right)+\nabla^{n} \\
& =-\boldsymbol{\phi} \times \boldsymbol{f}^{n}+\left(\boldsymbol{v}^{n} \times \boldsymbol{M}_{2}-\left(2 \boldsymbol{w}_{i e}^{n}+\boldsymbol{w}_{e n}^{n}\right) \times\right) \delta \boldsymbol{v}^{n}+\boldsymbol{v}^{n} \times\left(2 \boldsymbol{M}_{1}+\boldsymbol{M}_{3}\right) \delta \boldsymbol{p}+\nabla^{n} \\
& =-\boldsymbol{\phi} \times \boldsymbol{f}^{n}+\boldsymbol{M}_{4} \delta \boldsymbol{v}^{n}+\boldsymbol{M}_{5} \delta \boldsymbol{p}+\nabla^{n}
\end{aligned}
$$

Position error:

$$
\delta \dot{\boldsymbol{p}}=\boldsymbol{M}_{6} \delta \boldsymbol{v}^{n}+\boldsymbol{M}_{7} \delta \boldsymbol{p}
$$

among them,

$$
\begin{gathered}
\boldsymbol{M}_{1}=\left[\begin{array}{ccc}
0 & 0 & 0 \\
-w_{i e} \sin L & 0 & 0 \\
w_{i e} \cos L & 0 & 0
\end{array}\right], \boldsymbol{M}_{2}=\left[\begin{array}{ccc}
0 & -\frac{1}{R_{M}+h} & 0 \\
\frac{1}{R_{N}+h} & 0 & 0 \\
\frac{\tan L}{R_{N}+h} & 0 & 0
\end{array}\right] \boldsymbol{M}_{3}=\left[\begin{array}{ccc}
0 & 0 & \frac{v_{N}^{n}}{\left(R_{M}+h\right)^{2}} \\
0 & 0 & -\frac{v_{E}^{n}}{\left(R_{N}+h\right)^{2}} \\
\frac{v_{E}^{n} \sec ^{2} L}{R_{N}+h} & 0 & -\frac{v_{E}^{n} \tan L}{\left(R_{N}+h\right)^{2}}
\end{array}\right], \\
\boldsymbol{M}_{6}=\left[\begin{array}{ccc}
0 & \frac{1}{R_{M}+h} & 0 \\
\frac{\sec L}{R_{N}+h} & 0 & 0 \\
0 & 0 & 1
\end{array}\right]\left[\begin{array}{ccc}
0 & -\frac{v_{N}^{n}}{\left(R_{M}+h\right)^{2}} \\
\frac{v_{E}^{n} \sec L \tan L}{R_{M}+h} & 0 & -\frac{v_{E}^{n} \sec L}{\left(R_{N}+h\right)^{2}} \\
0 & 0 & 0
\end{array}\right]
\end{gathered}
$$

\subsection{SINS/OD Integrated Navigation Model}

Based on the analysis of the error model, a SINS/OD integrated navigation model is established.

As can be seen from the above analysis, odometer can inhibit the spread of pure INS errors, but its solution process reference $C_{b}^{n}$, This creates a cross-talk with SINS, Coupled with its own $\delta K_{D}$ affected by tire pressure, road conditions etc. decided that this combination cannot be unlimited to maintain high accuracy, you can predict the long-term error will increase. In order to prolong the high-precision retentivity of launch vehicle's vehicle orientation and orientation system under combat environment, the launch vehicle in the combat area must be equipped with the RFID positioning system according to the above scheme and assisted with SINS/OD positioning under no stopping conditions, Improve launch vehicle field combat mobility.

\section{RFID Positioning System Design}

\subsection{RFID Working Principle.}

RFID consists of a tag, a reader and an antenna. The tag stores digital information, and the reader controls the RF module to transmit the RF signal through the antenna and read the corresponding tag 
information as shown in Figure 1, RFID can identify high-speed moving objects, and identify tags through the plastic and other materials and can identify multiple tags[10].

\subsection{Design of Launch Vehicle Using RFID Positioning Scheme.}

Tags are divided into passive tags and active tags. In view of combat use, choose passive tags with encryption processing. readers can use high-speed readers, which cost $\mathrm{t} 0=20 \mathrm{~ms}$ to identify tags and read the information. Recognition distance can be adjusted by changing frequency,according to the needs of use. the recognition distance between $1 \mathrm{~m} \sim 50 \mathrm{~m}$ are optional.

(1) method No.1

Radio frequency tags are laid on the center of the lane surface. The tags store the geographical information code of the location (latitude, longitude, elevation, etc.). The reader is installed at the bottom of the launch vehicle. When the vehicle passes the tag, the information is read and sent to the onboard computer and compared with other positioning system information. Taking the rolling damage and other issues into account, sink the tags or paste them in the road reflector button, as shown in Figure 2.

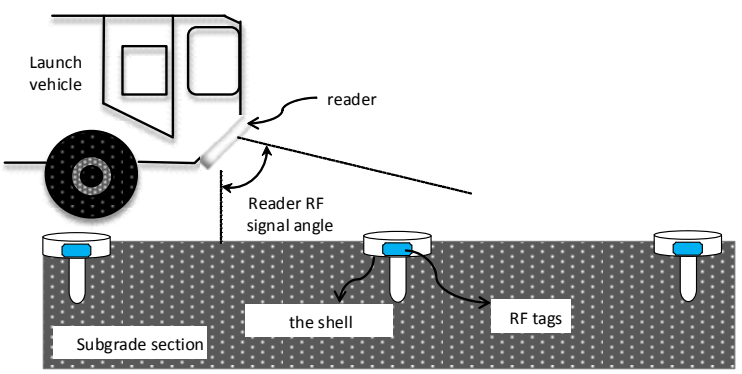

Fig. 2 Driveway installation of RFID positioning schematic diagram

After several parameter adjustment, the program driving test shows that once identified, the accuracy of positioning accuracy can reach $0.5 \mathrm{~m}$, fully meet the requirement of moving-positioning correction.And it easy to use without parking. If drive at a speed of about $30 \mathrm{~km} / \mathrm{h}$, single-point recognition is better.

(2) method No.2

The RF tags are installed equidistantly on the isolation road in the road or on the roadside protection pile. The reader is installed on the left (right) side of the launch vehicle and the tags are installed at the same hight. The position information is read during the moving, which is called the roadside sign positioning as Figure 3 shows.

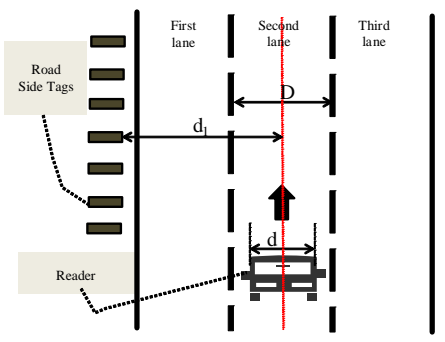

Fig. 3 Roadside installation of RFID positioning schematic diagram

$D$ is the width of the lane, $d$ is the launch vehicle width, $d 1$ is the vertical distance corresponding to the lane centerline, and the label stores the coordinate code of the position of the lane corresponding to the lane centerline. According to the survey of drivers who have special vehicles driving experience, $100 \%$ of respondents said that driving a vehicle in a single lane is very easy. Based on it, the positioning error analysis is carried out for the practical conditions.

As can be seen from Figure 3, On the condition of the launch vehicle maintain in a lane, the horizontal theoretical positioning error is:

$$
\boldsymbol{\delta} x=\frac{D-d}{2} .
$$

Longitudinal error is determined by recognition distance, recognition angle and vehicle speed. 


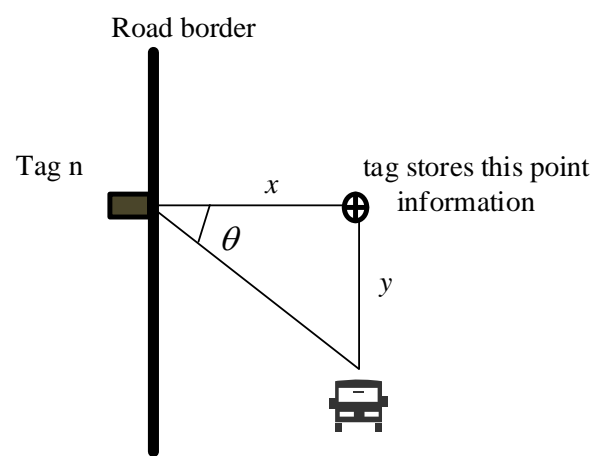

Fig. 4 The longitudinal position error

$\mathrm{x}, \mathrm{y}$ are the horizontal distance and vertical distance when the RF tag receives the RF signal from the reader, $\theta$ is the angle at which the tag receives the signal. so the longitudinal error is:

$$
\boldsymbol{\delta} y=x \tan \theta-v \cdot t_{0}
$$

$v$ is the vehicle speed, $t_{0}$ is the time of radio frequency identification.

Then the positioning coordinate point probability deviation is:

$$
\boldsymbol{\delta} r=\sqrt{\boldsymbol{\delta} x^{2}+\boldsymbol{\delta} y^{2}}
$$

According to the national road standards, the width of the single lane of the first-class and fourth-class roads is about $3.5 \mathrm{~m}$, for example, the freeway lanes's wide are $3.75 \mathrm{~m}$. According to Russia's "Poplar" launch vehicle standards, assume that the car width is $3 \mathrm{~m}$, the receiving signal angle is $15^{\circ}$, driving speed is of $40 \mathrm{~km} / \mathrm{h}$ in the second lane:

$\delta x \doteq 0.375 \mathrm{~m}, \delta y \doteq 1.285 \mathrm{~m}$

The theoretical calculation accuracy is:

$\boldsymbol{\delta} r=1.338 \mathrm{~m}$

The result can reach the magnitude of US military GPS.

In actual engineering application, by adjusting the identification distance and power of radio frequency, the identification angle can be adjusted without calculating the reaction distance under the normal driving speed of $20 \mathrm{~km} / \mathrm{h} \sim 50 \mathrm{~km} / \mathrm{h}$, because the measurement cannot be performed in the field experiment as long as the measurement The difference between the actual position and the theoretical position of the vehicle when the tag is recognized. Field experiments were conducted on a section of abandoned expressway with the interval of $10 \mathrm{~m}$ in a total length of $300 \mathrm{~m}$. Radio frequency identification parameters were rationally adjusted and allocated. The experimental car width is 2.5 m.Respectively, drive at a speed of $20 \mathrm{~km} / \mathrm{h}, 30 \mathrm{~km} / \mathrm{h}, 50 \mathrm{~km} / \mathrm{h}$ into the recognition area, the DGPS positioning data as a reference, the effective recognition rate of $97.7 \%$.So use 24 experimental positioning data of each group for analysis, the result is as follows.

Table.1 Positioning deviation under different speed

\begin{tabular}{ccc}
\hline Groups & Speed,km/h, & Average positioning deviation, $\mathrm{m}$, \\
\hline 1 & 20 & 1.96 \\
2 & 30 & 1.65 \\
3 & 50 & 2.42 \\
\hline
\end{tabular}

\section{SINS /OD/RFID Combined Location Model}

\subsection{Design}

The research group proposed the idea of "moving the satellite to the ground and letting the static landmarks move", and made use of the RFID location to make up for the shortcomings of the traditional modes__ satellites being damaged in wartime and the parking positioning restricted by maneuver.

The scheme design is as follows: vehicle SINS/OD travels within the theater according to the planned operation plan, and does not have to travel deliberately to the area where the passive RFID 
tag is arranged. However, By the way passing a RFID positioning area, aidding correcting SINS/OD, so as to reduce the error.

\subsection{Establish SINS/OD/RFID Combination Model}

\subsubsection{System Design}

Both OD and RFID fixes provide Kalman filter observations, giving two different error corrections for SINS state quantities. The observation precision is different, the covariance matrix is different, and the sampling frequency of RFID positioning correction is much lower than OD, resulting in the observation of the input filter with time asynchronism. This problem can be solved with Paralle-Kalman Filter (PKF).

As shown in Fig.5, OD has strong observability for adding table, gyro and attitude error, and can simultaneously correct speed, position, attitude, table and gyro; RFID positioning correction point only provides absolute position coordinates, Outside the position, the remaining observability is weak, but the accuracy of the observations is high, $0 \sim 2 \mathrm{~m}$,.System set breakpoints, when collected OD or RFID positioning data, automatically determine the appropriate choice of filter for navigation correction. Among them, SINS height channel divergence is suppressed by on-board barometric altimeter. RFID positioning information includes latitude, longitude, elevation and gravitational acceleration, and the elevation error is almost zero, which is directly used to replace solution calculation value.

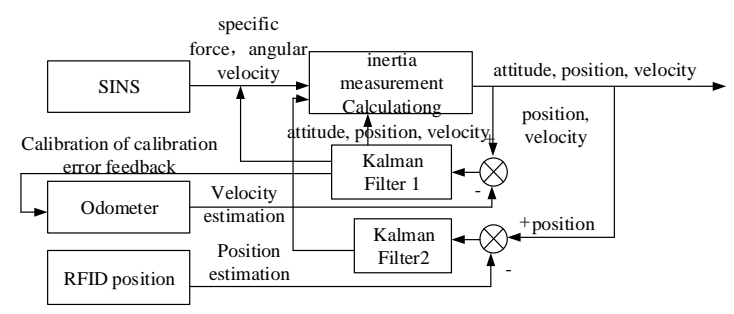

Fig.5 SINS/OD/RFID integrated navigation principle block diagram

\subsubsection{System State Equation}

System status is:

$$
\boldsymbol{X}(t)=\left[\begin{array}{lllllllllllll}
\delta v_{E} & \delta v_{N} & \phi_{E} & \phi_{N} & \phi_{U} & \delta \lambda & \delta L & \nabla_{x} & \nabla_{y} & \varepsilon_{x} & \varepsilon_{y} & \varepsilon_{z} & \delta K_{D}
\end{array}\right]^{\mathrm{T}}
$$

System state equation is:

$$
\dot{\boldsymbol{X}}(t)=\boldsymbol{F}(t) \boldsymbol{X}(t)+\boldsymbol{W}(t)
$$

The system noise vector is a zero-mean white noise vector with variance $Q$. System status matrix is $13 \times 13$ matrix:

The components are as follows:

$$
\boldsymbol{F}(\mathrm{t})=\left[\begin{array}{llllll}
\boldsymbol{F}_{11} & \boldsymbol{F}_{12} & \boldsymbol{F}_{13} & \boldsymbol{N}_{1} & \boldsymbol{0}_{2 \times 3} & \\
\boldsymbol{F}_{21} & \boldsymbol{F}_{22} & \boldsymbol{F}_{23} & \boldsymbol{0}_{3 \times 2} & \boldsymbol{N}_{2} & \boldsymbol{0}_{7 \times 1} \\
\boldsymbol{F}_{31} & \boldsymbol{F}_{32} & \boldsymbol{F}_{33} & \boldsymbol{0}_{2 \times 3} & \boldsymbol{0}_{2 \times 2} & \\
& \boldsymbol{0}_{6 \times 7} & & & \boldsymbol{\alpha} &
\end{array}\right]
$$

$$
\boldsymbol{F}_{11}=\left[\begin{array}{cc}
\frac{v_{N}^{n} \tan L}{R_{N}+h} & 2 w_{i e} \sin L+\frac{v_{E}}{R_{N}+h} \tan L \\
-2 w_{i e} \sin L-\frac{v_{E}}{R_{N}+h} \tan L & 0
\end{array}\right], \boldsymbol{F}_{23}=\left[\begin{array}{cc}
0 & 0 \\
0 & -w_{i e} \sin L \\
0 & w_{i e} \cos L+\frac{v_{E}^{n}}{R_{N}+h} \sec ^{2} L
\end{array}\right], \boldsymbol{N}_{2}=-\boldsymbol{C}_{b}^{n},
$$




$$
\begin{aligned}
& \boldsymbol{F}_{31}=\left[\begin{array}{cc}
0 & \frac{1}{R_{M}+h} \\
\frac{\sec L}{R_{N}+h} & 0
\end{array}\right], \boldsymbol{F}_{32}=\boldsymbol{O}_{2 \times 3}, \boldsymbol{F}_{33}=\left[\begin{array}{cc}
0 & 0 \\
0 & \frac{v_{E}^{n} \tan L \sec L}{R_{N}+h}
\end{array}\right], \boldsymbol{N}_{1}=\left[\begin{array}{ll}
\boldsymbol{C}_{b 11}^{n} & \boldsymbol{C}_{b 12}^{n} \\
\boldsymbol{C}_{b 21}^{n} & \boldsymbol{C}_{b 22}^{n}
\end{array}\right], \\
& \boldsymbol{\alpha}=\operatorname{diag}\left\{\begin{array}{llllll}
-\frac{1}{\tau_{G E}} & -\frac{1}{\tau_{G N}} & -\frac{1}{\tau_{A E}} & -\frac{1}{\tau_{A N}} & -\frac{1}{\tau_{A U}} & -\frac{1}{\tau_{D}}
\end{array}\right\}
\end{aligned}
$$

Among them, $\delta v_{N}, \delta v_{N}$ is the east and north of the speed error , $\phi_{E}, \phi_{N}, \phi_{U}$ is the attitude misalignment angle; SINS latitude and longitude error are $\delta \lambda, \delta L$; gyro drift are $\varepsilon_{x}, \varepsilon_{y}, \varepsilon_{z}$, add table zero bias $\nabla_{x}, \nabla_{y}$. Due to the long duration of flight, the description of the model errors of accelerometers, gyroscopes and odometers, an additional stochastic first-order Markov process deviation model is used, and the inverse correlation time respectively is $-\frac{1}{\tau_{A}},-\frac{1}{\tau_{G}},-\frac{1}{\tau_{D}}$.

\subsubsection{System Observation Equation}

SINS / OD filter observation equation is:

$$
\boldsymbol{Z}_{\mathrm{OD}}(t)=\boldsymbol{H}_{\mathrm{OD}}(t) \boldsymbol{X}(t)+\boldsymbol{V}_{\mathrm{OD}}(t)
$$

$\boldsymbol{V}(t)$ is a measurement noise vector with zero mean variance $\boldsymbol{R}_{\mathrm{OD}}$.

Observations $\boldsymbol{Z}_{\mathrm{OD}}(t)$ are calculated as follows,

$$
\boldsymbol{Z}_{\mathrm{OD}}(t)=\hat{\boldsymbol{v}}^{n}-\hat{\boldsymbol{v}}_{D}^{n}=\delta \boldsymbol{v}^{n}-\boldsymbol{v}_{D}^{n} \times \phi-\boldsymbol{v}_{D}^{n} \delta K_{D}
$$

The measurement matrix $\boldsymbol{H}(t)$ expands as follows,

$$
\boldsymbol{H}(t)=\left[\begin{array}{ccccccc}
1 & 0 & 0 & v_{D U}^{n} & -v_{D N}^{n} & \boldsymbol{0}_{1 \times 7} & -v_{D E}^{n} \\
0 & 1 & -v_{D U}^{n} & 0 & v_{D E}^{n} & \boldsymbol{0}_{1 \times 7} & -v_{D N}^{n}
\end{array}\right]
$$

SINS/RFID filter measurement equation is,

$$
\begin{gathered}
\boldsymbol{Z}_{\mathrm{RFID}}(t)=\boldsymbol{H}_{\mathrm{RFID}}(t) \boldsymbol{X}(t)+\boldsymbol{V}_{\mathrm{RFID}}(t) \\
\boldsymbol{Z}_{\mathrm{FRID}}(t)=\left[\begin{array}{l}
\lambda_{\text {SINS }}-\lambda_{\text {RFID }} \\
L_{\text {SINS }}-L_{R F I D}
\end{array}\right]=\left[\begin{array}{l}
\delta \lambda-V_{R F I D 1} \\
\delta L-V_{R F I D 2}
\end{array}\right]
\end{gathered}
$$

Analysis of the above RFID positioning results,the variance of observation noise $\boldsymbol{R}_{\mathrm{FRID}}$ is chosen as $\boldsymbol{R}_{\lambda}=(1.2 m)^{2}, \boldsymbol{R}_{L}=(1.2 m)^{2}$,

\section{Vehicle Experiment and Analysis}

In order to verify the combined positioning effect of RFID-assisted SINS/OD long-range navigation, a moving vehicle experiment up to 3.6 hours was carried out with a travel of about 300 $\mathrm{km}$.Experimental vehicle positioning and orientation system parameters are as follows: Strapdown inertial navigation system error is: gyro constant drift $0.01^{\circ} / \mathrm{h}$, the accelerometer constant drift $0.1 \mathrm{mg}$, calibration OD scale coefficient error is 0.0131. In the experiment, the actual application is simulated. it is assumed that the odometer is damaged during the last phase.After a long navigation (about $1 \mathrm{~h}$ ), RFID positioning was introduced at randomized intervals during driving, and the positioning deviation was reasonably determined to be $1.2 \mathrm{~m}$. Positioning benchmarks in the process of driving provided by the car GPS. 


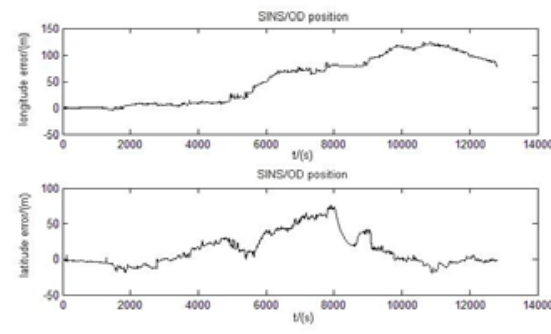

Fig.6 SINS/OD positioning errors

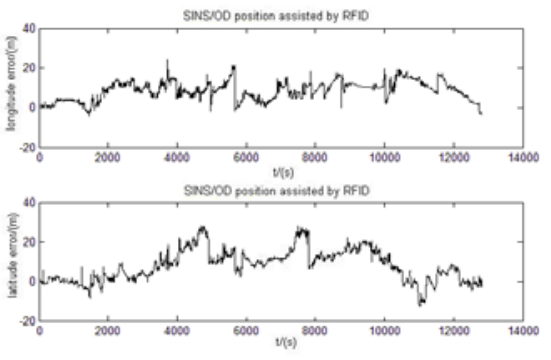

Fig.7 Positioning errors revised by RFID

Analysis of Fig.6 shows that the SINS / OD combination can maintain a higher positioning result for a longer time. If the reference precision of $20 \mathrm{~m}$ is required, then the condition can be maintained about $1.5 \mathrm{~h}$, then the error gradually diverges, even after the odometer is analog damaged, the positioning error is more than $100 \mathrm{~m}$.

If the vehicle passes through four RFID positioning areas at $5000 \mathrm{~s}, 5800 \mathrm{~s}, 7900 \mathrm{~s}$, and $10000 \mathrm{~s}$ according to the usage conditions and obtains the correction information, the result is shown in Fig.7, the positioning error will be greatly reduced and the error will be controlled at Accuracy within the required range, reaching a high level. The integrated positioning doubles the SINS/OD navigation time, up to 3.6 h or more.

\section{Conclusion}

Under the background of the current application of launching vehicle positioning technology in the field of combat applications, this paper discusses and proposes the application of RFID positioning to correct the SINS/OD scheme.Designing and verifying the RFID moving positioning method under the engineering background, and concluding that the positioning accuracy is high and easy to realize. Meanwhile, in order to solve the problem that SINS/OD precision does not meet the requirements after a long navigation, the experimental results based on SINS/OD/RFID model show that SINS / OD with four random RFID positioning aids to suppress the error divergence and extend the navigation time to meet the accuracy requirements Doubling the launching vehicle to enhance the ability of combat capability in the field, providing some reference for the realization of the project.

\section{References}

[1]. Chen Si, Zhong Qiyuan, Tan Zhilong, et al.An overview of vehicle positioning and orientation technology [J]. Cruise Missile, Vol. 10 (2017), p. 26-30.

[2]. Ningbo, Wang Xiaqing, Huang Xianghuan. Development and Application of Military Vehicle Location and Navigation System [J]. Technical Prospect, Vol. 31 (2015), p. 205-206.

[3]. Li Rui-Tao, Xu Sheng-Hong, Cao Wen-Jing.A Modification of Vehicle Inertial Attitude Based on Landmark Information [J]. Journal of Naval Air Engineering, Vol.29 (2014) p. 315-318.

[4]. J.H. Wang, Y. Gao. GPS-based land vehicle navigation system assisted by a low-cost gyro-free INS using neural network [J]. Journal of Navigation, Vol. 57 (2004), p. 417-428.

[5]. [5] Fang Weijun.GM and Digital Map Matching for Integrated Navigation [D]. Nanjing: Nanjing University of Aeronautics and Astronautics, 2009.

[6]. Yan Gongmin. Vehicle autonomous positioning system [D]. Xi'an: Northwestern Polytechnical University, 2006.

[7]. Tang Yong, Wang Minghui, Wang Chunfeng RFID technology and application [M]. Zhenjiang: Jiangsu University Press, 2014.11, p.1-7

[8]. Wang Kai.Compositional Kalman Filter Based on Combination Orientation and Direction Technology [D]. Xi'an: Rocket Military Engineering University, 2012. 
[9]. Qin Yongyuan. Inertial Navigation (Second Edition) [M]. Beijing: Science Press, 2014, p. 311-316.

[10]. Peng Li. Radio frequency identification (RFID) technology foundation [M] Beijing: Beijing University of Aeronautics and Astronautics Press, 2012.9, p.1-2 